\author{
Jen-San Chen ${ }^{1}$ \\ Professor \\ e-mail: jschen@ccms.ntu.edu.tw \\ Der-Wei Chang \\ Graduate Student \\ Department of Mechanical Engineering, \\ National Taiwan University, \\ Taipei, Taiwan 10617
}

\title{
Snapping of a Shallow Arch With Harmonic Excitation at One End
}

In this paper we demonstrate both numerically and experimentally that it is possible to make a pinned-pinned shallow arch snap to and remain vibrating on the other side by harmonic excitation in the longitudinal direction at the end. One end of the arch is fixed in space, while the other end is attached to a mechanical shaker via a spring. The shaker-mount is first moved a small distance toward the arch to ensure that the arch assembly possesses two stable equilibrium positions, one on each side of the base line. The spring connecting the arch end and the mechanical shaker is carefully chosen such that a small shaker stroke can induce a large vibration amplitude in the arch. The natural frequencies of the two (initial and snapped, respectively) positions are measured first. By adjusting the excitation frequency of the mechanical shaker to the first natural frequency of either position of the arch, we demonstrate that the arch can be snapped to and remain vibrating on the other side when the magnitude of the electric current flowing through the shaker is properly chosen. The vibrant snapping action of the arch recorded in the experiment is confirmed by numerical simulation. [DOI: 10.1115/1.2748479]

\section{Introduction}

Historically, the interest in shallow arch research is primarily on the snap-through buckling when the arch is under lateral loading. The first theoretical prediction on the static critical load was conducted by Timoshenko in 1935 [1], in which a pinned sinusoidal arch was subject to a uniformly distributed load. Timoshenko's pioneering work was followed and extended by many other researchers on various topics, including the snap-through phenomenon under dynamic load. The first theoretical prediction of dynamic buckling load was conducted by Hoff and Bruce in 1954 [2], in which they studied the stability of a sinusoidal arch under unit step loading and ideal impulsive loading. A good introduction on the subject can be found in Simitses' book [3]. More new publications on the dynamic snap-through buckling of shallow arches can be found in a recent paper by the first author [4].

Besides the quasi-static, impulsive, and step loadings discussed above, the dynamic response of a shallow arch under periodic excitation has also been studied by some researchers. Previous research in this regard may be roughly divided into two groups. The first group focuses on smaller excitation and vibration amplitude with emphasis on the nonlinear response such as superharmonic, subharmonic, and internal resonances. Thomsen [5] studied the chaotic vibration of a circular high arch loaded by a harmonic force at its crown, a problem initiated by Bolotin in his book [6]. Tien et al. [7,8] and Bi and Dai [9] used a two-term approximation to study the internal resonance of a shallow arch under lateral loading with both averaging method and direct integration method. With the same two-term approximation, Malhotra and Sri Namachchivaya $[10,11]$ also investigated the possibility of chaotic response when the shallow arch is under 1:1 and 1:2 resonances. Chen and Yang [12] studied, both experimentally and theoretically, the nonlinear response of a shallow arch under harmonic excitation at one end.

The second group considers the case when the vibration amplitude is so large that dynamic snap-through occurs. The critical load amplitude and frequency of an arch under harmonic excitation is harder to predict compared to the problem of step or impulsive loading. Huang [13] used a cycle-averaging approach to

\footnotetext{
${ }^{1}$ Corresponding author.

Contributed by the Technical Committee on Vibration and Sound of ASME for publication in the Journal OF VIBRATION AND ACOUSTICS. Manuscript received December 23, 2006; final manuscript received April 16, 2007. Review conducted by Jean $\mathrm{Zu}$.
}

predict the critical load when the excitation frequency is very high. Plaut and Hsieh [14] used a one-term approximation to numerically study the critical load when the arch is subject to a two-frequency excitation. Blair et al. [15] simplified the arch with a two-rigid-link model and used the harmonic balance method to study its dynamic response when the linkage is under harmonic excitation.

From the above literature review, we notice that while some theoretical investigations on the dynamic response of a periodically excited shallow arch exist, experimental investigation is relatively rare. More precisely, no experimental work can be found in the literature when the periodic force is applied in the lateral direction. Part of the reason is due to the difficulty in implementing a pulsating load with constant amplitude in the laboratory, especially when the vibration amplitude becomes large during snapping. In the case when the periodic external load is in the longitudinal direction, on the other hand, it becomes relatively easier to realize, as reported by Chen and Yang [12]. However, Chen and Yang's experimental setup allows nonlinear vibration of the shallow arch only in the neighborhood of the initial unstressed position. They were unable to snap the arch to the other side with the shaker directly attached to the end. This is because when the arch snaps to the other side, the end movement of the arch will exceed the stroke limit of the attached shaker.

In this paper, we propose an experimental setup that permits us to make the arch snap and remain vibrating on the other side with an electro-mechanical shaker attached to one end. The experimental setup consists of an additional spring between the shaker and the arch end. The shaker-mount is first moved a small distance toward the arch to ensure that the arch assembly possesses two stable equilibrium positions before harmonic excitation. The parameter of the additional spring is chosen such that small shaker stroke can induce large vibration amplitude of the arch. By doing so we demonstrate that the attached shaker can snap the arch to either side at will, by adjusting the excitation frequency and amplitude. Experimental observation is verified by theoretical prediction.

\section{Experimental Setup}

Figure 1 is a schematic diagram of the experimental setup. The arch is made of a brass strip with Young's modulus $101 \mathrm{GPa}$ and mass density $8840 \mathrm{~kg} / \mathrm{m}^{3}$. The length $L$ of the arch is $44 \mathrm{~cm}$ and 


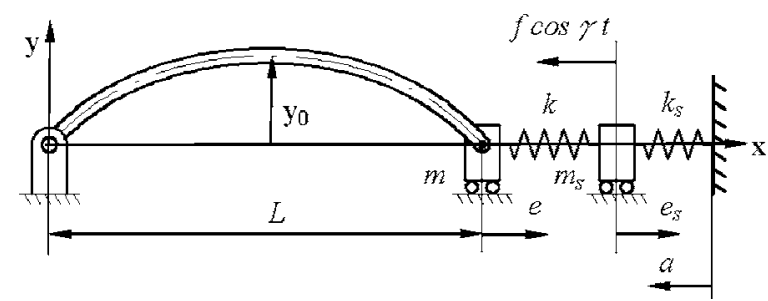

Fig. 1 Schematic diagram of a shallow arch with one end attached to a mechanical shaker. The shaker-mount is moved toward the arch a distance $a$ to induce initial compression in the arch.

the cross section is $20 \mathrm{~mm} \times 1.0 \mathrm{~mm}$. The brass strip is carefully bent into a half-sinusoidal shape with the initial height $h$ at the middle point, or mathematically,

$$
y_{0}=h \sin \frac{\pi x}{L}
$$

In the experiment, $h$ is equal to $14 \mathrm{~mm}$. Both ends of the curved beam are attached to roller bearings to simulate a pinned condition. One end of the arch is fixed in space, while the other end is attached to a slider and is allowed to slide on a guiding rod. The slider mass is denoted by $m$, and is weighed to be $0.9 \mathrm{~kg}$. The slider is attached in series to a spring $k$, which is then attached to the central core of the mechanical shaker.

The mechanical shaker can be modeled as a mass-spring system with parameters $m_{s}$ and $k_{s}$. The parameters $m_{s}$ and $k_{s}$ of the shaker can be determined experimentally to be $m_{s}=0.158 \mathrm{~kg}$ and $k_{s}$ $=12.8 \mathrm{kN} / \mathrm{m}$ [16]. The magnetic force on the central core of the shaker is controlled by the electric current flowing into the shaker. A power amplifier connected to the shaker is responsible for pumping electric current proportional to the harmonic voltage signal from a function generator. With this arrangement, the magnetic force on the central core of the shaker can be estimated. The lateral speeds at the middle point of the arch and the longitudinal motions of the end slider and shaker can be measured by a laser Doppler vibrometer (LDV) system. The speed signals from the LDV system can be integrated to obtain the displacement history.

The main difference between the current experimental setup and the one presented in [12] is the additional spring $k$. It is noted that the selected shaker has a stroke limit about $0.5 \mathrm{~mm}$ when it is attached to the arch directly. However, when the curved beam is pulled from the initial curved shape to the straight position, the end moves about $1 \mathrm{~mm}$. Therefore, with the original design in [12] it is difficult to snap the arch to the other side. The reason the shaker has a small stroke limit when the shaker is attached to the slider directly is because the mechanical impedance of the archslider system in the original design is high as seen from the shaker. Therefore, the shaker needs large force in order to move the arch slider. As a consequence, more current needs to be pumped into the shaker. There is a protective circuit in the power amplifier which limits the amount of current allowed to flow into the amplifier. This protective circuit limits the shaker stroke of the original design. By adding an additional soft spring between the arch slider and the shaker as in the current design, the mechanical impedance as seen from the shaker can be effectively reduced. In a later section we will explain how to determine the additional parameter $k$ such that small mechanical shaker movement can induce large amplitude vibration in the arch.

One question may naturally arise at this point; i.e., Why not simply find a more powerful shaker which has a longer stroke? With a more powerful shaker, it is indeed easier to snap the arch to the other side. However, the arch will snap back immediately and continue snapping back and forth between the two positions. As a consequence, it is almost impossible to control the arch between the two positions as desired.

\section{Equations of Motion}

Consider the theoretical model shown in Fig. 1. The mass $m_{s}$ of the central core of the shaker is subject to a harmonic excitation force $f \cos \gamma t$, where $f$ and $\gamma$ are the amplitude and the frequency of the external force. The initial (unstressed) and deformed shapes of the arch are $y_{0}$ and $y$, respectively, both measured from the same base line. The equation of motion of the arch can be written as

$$
\rho A y_{, t t}=-E I\left(y-y_{0}\right)_{, x x x x}+p y_{, x x}
$$

The parameters $E, \rho, A$, and $I$ are Young's modulus, mass density, area, and area moment of inertia of the cross section of the arch, respectively. $p$ is the axial force in the deformed arch. The mount of the shaker is moved from the initial position a small distance $a$ toward the arch to induce certain initial compression in the assembly. The purpose of this initial shaker-mount movement $a$ is to ensure the existence of two stable positions of the assembly. If there exists only one stable equilibrium position, then there is no point trying to snap the arch to the other side. The force balances of the end slider $m$ and the central core $m_{s}$ of the shaker give two additional equations:

$$
\begin{gathered}
m_{s} \ddot{e}_{s}=k\left(e-e_{s}\right)-k_{s}\left(e_{s}+a\right)+f \cos \gamma t \\
m \ddot{e}=-k\left(e-e_{s}\right)-p
\end{gathered}
$$

$e$ and $e_{s}$ are the motion of the end slider and the central core of the shaker, respectively; both are measured from the initial unstressed configuration before shaker movement $a$. The relation between $e$ and the shape change of the arch can be established from the elastic extensibility of the arch

$$
p=\frac{E A}{L}\left\{e+\frac{1}{2} \int_{0}^{L}\left[\left(y_{, x}\right)^{2}-\left(y_{0, x}\right)^{2}\right] d x\right\}
$$

Equations (2)-(5) can be nondimensionalized by introducing the following dimensionless parameters (with asterisks):

$$
\begin{gathered}
\left(y^{*}, y_{0}^{*}, h^{*}\right)=\frac{1}{r}\left(y, y_{0}, h\right), \quad x^{*}=\frac{\pi x}{L}, \quad t^{*}=\frac{\pi^{2} t}{L^{2}} \sqrt{\frac{E I}{A \rho}}, \\
\left(\gamma^{*}, \omega^{*}\right)=\frac{L^{2}}{\pi^{2}} \sqrt{\frac{A \rho}{E I}}(\gamma, \omega), \quad\left(p^{*}, f^{*}\right)=\frac{L^{2}}{\pi^{2} E I}(p, f), \\
\left(e_{s}^{*}, e^{*}, a^{*}\right)=\frac{L}{\pi^{2} r^{2}}\left(e_{s}, e, a\right), \quad\left(m_{s}^{*}, m^{*}\right)=\frac{I \pi^{4}}{L^{3} A^{2} \rho}\left(m_{s}, m\right), \\
\left(k_{s}^{*}, k^{*}\right)=\frac{L}{E A}\left(k_{s}, k\right), \quad \mu^{*}=\frac{L^{2} \mu}{\pi^{2} \rho A r} \sqrt{\frac{\rho}{E}}
\end{gathered}
$$

The parameters $\mu$ and $\omega$ are the damping and natural frequency, respectively, of the arch-shaker system, which will be discussed later. $r$ is the radius of gyration of the cross section of the arch. After substituting the above relations into Eqs. (2)-(5), and dropping all the superposed asterisks thereafter for simplicity, we obtain the three dimensionless equations of motion:

$$
\begin{gathered}
y_{, t t}=-\left(y-y_{0}\right)_{, x x x x}+\left\{e+\frac{1}{2 \pi} \int_{0}^{\pi}\left[\left(y_{, x}\right)^{2}-\left(y_{0, x}\right)^{2}\right]\right\} y_{, x x} \\
m_{s} \ddot{e}_{s}=-\left(k_{s}+k\right) e_{s}+k e-k_{s} a+f \cos \gamma t \\
m \ddot{e}=-\frac{1}{2 \pi} \int_{0}^{\pi}\left[\left(y_{, x}\right)^{2}-\left(y_{0, x}\right)^{2}\right] d x-(1+k) e+k e_{s}
\end{gathered}
$$

The boundary conditions for $y$ at $x=0$ and $\pi$ are 


$$
\begin{aligned}
y(0)-y_{0}(0) & =y_{, x x}(0)-y_{0, x x}(0) \\
& =y(\pi)-y_{0}(\pi)=y_{, x x}(\pi)-y_{0, x x}(\pi)=0
\end{aligned}
$$

It is noted that in establishing the boundary conditions for the lateral displacement $y(x)$, we ignore the small longitudinal position change of the right-hand end.

The dimensionless version of Eq. (1), i.e., the initial shape of the arch before the introduction of the initial compression, can be written as:

$$
y_{0}=h \sin x
$$

It is assumed that the shape of the loaded arch can be expanded as

$$
y(t)=y_{0}+\sum_{n=1}^{\infty} \alpha_{n}(t) \sin n x
$$

After substituting Eqs. (11) and (12) into (7)-(9), multiplying Eq. (7) by $\sin n x$ and integrating from $x=0$ to $\pi$ (Galerkin's procedure), we obtain the equations governing $\alpha_{n}, e$, and $e_{s}$ as

$$
\begin{gathered}
\ddot{\alpha}_{1}=-\alpha_{1}-\left(h+\alpha_{1}\right)\left[e+\frac{1}{4}\left(\alpha_{1}^{2}+2 h \alpha_{1}+\sum_{j=2}^{\infty} j^{2} \alpha_{j}^{2}\right)\right] \quad(13) \\
\ddot{\alpha}_{n}=-n^{4} \alpha_{n}-n^{2} \alpha_{n}\left[e+\frac{1}{4}\left(\alpha_{1}^{2}+2 h \alpha_{1}+\sum_{j=2}^{\infty} j^{2} \alpha_{j}^{2}\right)\right] \quad n=2,3, \ldots
\end{gathered}
$$

$$
\begin{gathered}
m_{s} \ddot{e}_{s}=-\left(k_{s}+k\right) e_{s}+k e-k_{s} a+f \cos \gamma t \\
m \ddot{e}=k e_{s}-(1+k) e-\frac{1}{4}\left(\alpha_{1}^{2}+2 h \alpha_{1}+\sum_{j=2}^{\infty} j^{2} \alpha_{j}^{2}\right)
\end{gathered}
$$

The equilibrium positions after the shaker-mount movement $a$ can be determined from the nonlinear equations (13)-(16) by neglecting the terms on the left-hand sides. The coordinates corresponding to these equilibrium positions are denoted by $\alpha_{n}^{(s)}, e^{(s)}$, and $e_{s}^{(s)}$.

\section{Selection of Spring Constant $k$ and Shaker-Mount Movement $a$}

Two objectives are borne in mind when we determine the spring constant $k$ and the initial shaker-mount movement $a$. First of all, we have to ensure the existence of two stable equilibrium positions, one on each side of the base line. In general, this condition can be met by increasing the movement $a$. Second, we hope that small mechanical shaker oscillation $e_{s}$ can induce large slider vibration $e$ when the shaker excites the arch at the first natural frequency of the assembly. This condition may be met by requiring that in the mode shape corresponding to the first natural frequency of the assembly, the component $e_{s}$ must be as small as possible compared to $e$.

The equilibrium positions of the arch-shaker assembly in Fig. 1 after a specified shaker movement $a$ can be determined from Eqs. (13)-(16) by neglecting all the acceleration terms. It can be shown that there is either only one stable equilibrium position, or sometimes there can be two stable equilibrium positions. All the stable equilibrium positions involve only $\alpha_{1} \sin x$ in expansion (12), which are called one-mode solutions. The first stable position, denoted as $P_{0}$ [17], is the one near the initial unstressed position $y_{0}$, which always exists and is always stable. This position can be achieved by moving the shaker-mount the distance $a$ quasistatically. The other possible stable one-mode solution is on the other side of the base line, denoted as $P_{1}^{-}$[17], which exists only under certain conditions. All equilibrium positions involving more than one mode are unstable. The stability of these equilibrium positions can be determined by using the energy method as de-

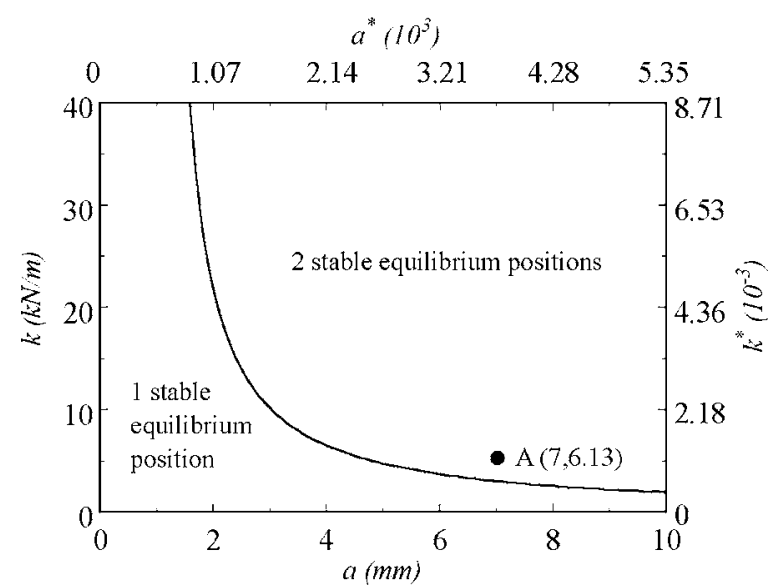

Fig. 2 The boundary separating the domains of two stable equilibrium positions and one stable equilibrium position in the $k$-a plane. Point A represents the design parameters we choose in our experimental setup.

scribed in the second author's thesis [16] and another paper published by the first author [17]. Based on the theory presented in $[16,17]$, we can determine the boundary of the parameter range in the $k$ - $a$ plane in Fig. 2 that guarantees the existence of two stable positions when the initial height $h=14 \mathrm{~mm}$ and shaker spring $k_{s}$ $=12.8 \mathrm{kN} / \mathrm{m}$ as in our experimental setup. For convenient reference, both the physical parameters (left and bottom) and the dimensionless ones (right and top) are used in the same figure. From this figure, we choose the initial shaker-mount movement as $7 \mathrm{~mm}$. With this initial mount movement, the spring constant $k$ has to be greater than $2.98 \mathrm{kN} / \mathrm{m}$.

To calculate the natural frequencies and the corresponding mode shapes of the assembly after the shaker movement $a$, we first linearize the nonlinear equations (13)-(16) in the vicinity of one of the two stable equilibrium positions $\alpha_{n}^{(s)}, e^{(s)}$, and $e_{s}^{(s)}$ by assuming

$$
\begin{gathered}
\alpha_{n}=\alpha_{n}^{(s)}+\widetilde{\alpha}_{n} \quad n=1,2,3, \ldots \\
e=e^{(s)}+\widetilde{e} \\
e_{s}=e_{s}^{(s)}+\widetilde{e}_{s}
\end{gathered}
$$

$\widetilde{\alpha}_{n}, \widetilde{e}$, and $\widetilde{e}_{s}$ are small perturbations superposed on the equilibrium positions. After substituting Eqs. (17)-(19) into Eqs. (13)-(16), linearizing with respect to $\widetilde{\alpha}_{n}, \widetilde{e}$, and $\tilde{e}_{s}$, we obtain the linearized equations of motion as

$$
\begin{aligned}
\ddot{\widetilde{\alpha}}_{1}= & -\widetilde{\alpha}_{1}-\widetilde{\alpha}_{1}\left(e^{(s)}+\widetilde{e}+\frac{1}{4}\left\{\left(\alpha_{1}^{(s)}\right)^{2}+2 \alpha_{1}^{(s)} \widetilde{\alpha}_{1}+2 h\left(\alpha_{1}^{(s)}+\widetilde{\alpha}_{1}\right)\right.\right. \\
& \left.\left.+\sum_{j=2}^{\infty} j^{2}\left[\left(\alpha_{1}^{(s)}\right)^{2}+2 \alpha_{j}^{(s)} \widetilde{\alpha}_{j}\right]\right\}\right)-\left(h+\alpha_{1}^{(s)}\right)\left\{\tilde{e}+\frac{1}{4}\left[2 \alpha_{1}^{(s)} \widetilde{\alpha}_{1}\right.\right. \\
& \left.\left.+2 h \widetilde{\alpha}_{1}+2 \sum_{j=2}^{\infty} j^{2} \alpha_{j}^{(s)} \widetilde{\alpha}_{j}\right]\right\}
\end{aligned}
$$




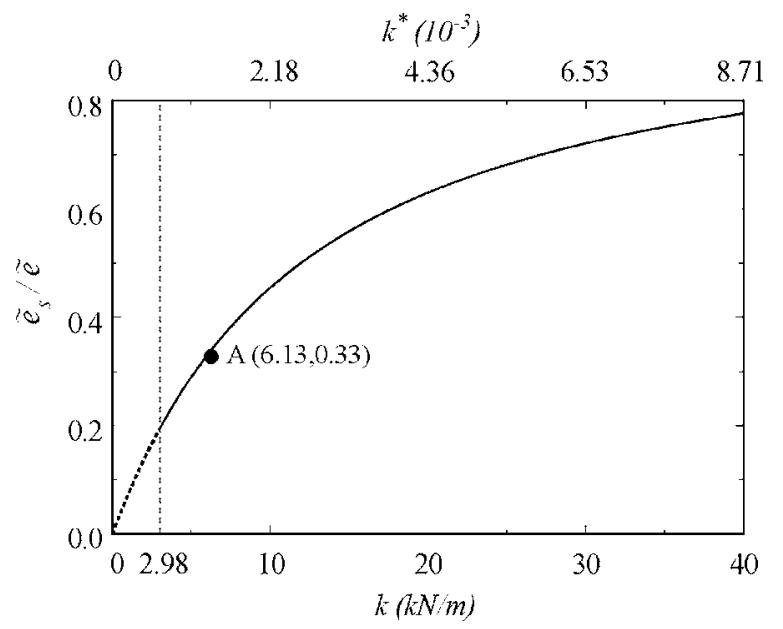

Fig. 3 The relation between spring constant $k$ and the ratio $\tilde{e}_{s} / \tilde{e}$. Only the spring constant $k$ on the right-hand side of the vertical dashed line can guarantee the existence of two stable equilibrium positions. Point $A$ represents the design parameters we choose in our experimental setup.

$$
\begin{aligned}
\ddot{\widetilde{\alpha}}_{n}= & -n^{4} \widetilde{\alpha}_{n}-n^{2} \widetilde{\alpha}_{1}\left(e^{(s)}+\tilde{e}+\frac{1}{4}\left\{\left(\alpha_{1}^{(s)}\right)^{2}+2 \alpha_{1}^{(s)} \widetilde{\alpha}_{1}+2 h\left(\alpha_{1}^{(s)}+\widetilde{\alpha}_{1}\right)\right.\right. \\
& \left.+\sum_{j=2}^{\infty} j^{2}\left[\left(\alpha_{j}^{(s)}\right)^{2}+2 \alpha_{j}^{(s)} \widetilde{\alpha}_{j}\right]\right\}-n^{2} \alpha_{n}^{(s)}\left\{\tilde{e}+\frac{1}{4}\left[2 \alpha_{1}^{(s)} \widetilde{\alpha}_{1}+2 h \widetilde{\alpha}_{1}\right.\right. \\
& \left.\left.+2 \sum_{j=2}^{\infty} j^{2} \alpha_{j}^{(s)} \widetilde{\alpha}_{j}\right]\right\} \quad n=2,3, \ldots \\
& m_{s} \ddot{\tilde{e}}_{s}=-\left(k_{s}+k\right) \widetilde{e}_{s}+k \widetilde{e} \\
& m \ddot{\widetilde{e}}=k \widetilde{e}_{s}-(1+k) \widetilde{e}-\frac{1}{2}\left[\alpha_{1}^{(s)} \widetilde{\alpha}_{1}+h \widetilde{\alpha}_{1}+\sum_{j=2}^{\infty} j^{2} \alpha_{j}^{(s)} \widetilde{\alpha}_{j}\right]
\end{aligned}
$$

For simplicity, at this stage we use only one term in the expansion (12). With the three linearized equations in terms of $\widetilde{\alpha}_{1}, \widetilde{e}$, and $\widetilde{e}_{s}$, we can calculate the first natural frequency and the associated mode shape in the neighborhood of position $P_{0}$ or $P_{1}^{-}$. We first choose $a=7 \mathrm{~mm}$ and focus our attention on the mode shape near $P_{0}$, then the relation between the ratio $\widetilde{e}_{s} / \widetilde{e}$ and $k$ can be plotted as in Fig. 3. As shown in Fig. 3, smaller stiffness $k$ can produce smaller ratio $\widetilde{e}_{s} / \widetilde{e}$. The vertical dashed line in Fig. 3 represents the lower bound of $k$, below which the arch possesses only one equilibrium position. We next focus our attention on the mode shape near $P_{1}^{-}$. The same analysis gives us another curve which is indistinguishable from the curve plotted above for position $P_{0}$. In other words, the spring chosen for the minimum $\tilde{e}_{s} / \widetilde{e}$ ratio near the position $P_{0}$ will also do the same near $P_{1}^{-}$. From these analyses, we expect that the vibration amplitude of the shaker can be only about one-third of the arch slider vibration when the arch is excited near positions $P_{0}$ and $P_{1}^{-}$at the respective first natural frequency. In other words, a small shaker vibration can now induce a large arch vibration. Whether this design indeed serves our purpose will be examined in the experiment later.

We select a spring with constant $k=6.13 \mathrm{kN} / \mathrm{m}$ for our experiment. The design parameters $a$ and $k$ are indicated by point " $\mathrm{A}$ " in Figs. 2 and 3. The photograph of our final experimental setup is shown in Fig. 4. Figure 4(a) shows the initial setup before the shaker-mount movement. In Fig. 4(b), the shaker-mount has been moved toward the arch ends a distance $a$. The arch is apparently

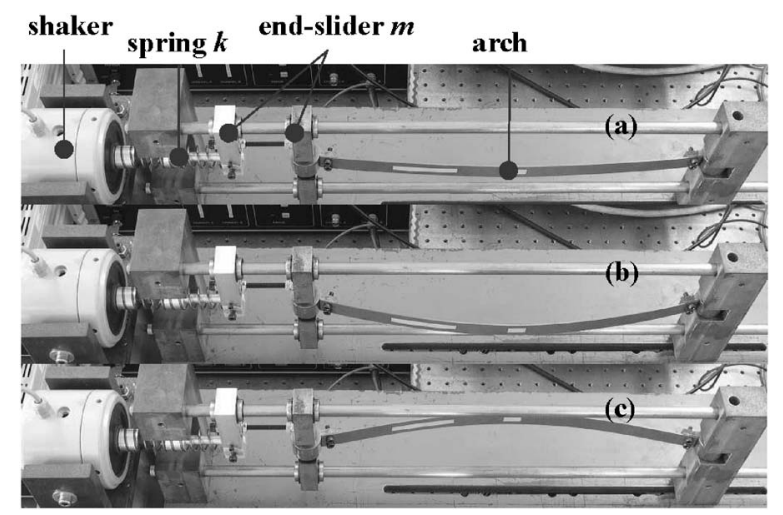

Fig. 4 Photograph of the experimental setup. (a) The initial unstressed position before the shaker-mount movement. (b) The $P_{0}$ position after the shaker-mount movement. The arch is bent more compared to (a). (c) The snapped position $P_{1}^{-}$indeed exists.

bent more compared to the initial shape in Fig. 4(a). Figure 4(c) demonstrates that the snapped position $P_{1}^{-}$indeed exists.

\section{Natural Frequencies and Damping Ratio of the Assembly}

One way to check whether the theoretical model of the assembly is correct is to compare the theoretical natural frequencies calculated from Eqs. (20)-(23) with those observed experimentally. In the calculation of the natural frequencies, we use three modes in the expansion (12). In the experiment, we measure the frequency response function of the assembly with the force on the central core of the shaker as the input and the lateral velocity at the middle point of the arch as the output. Figures 5(a) and 5(b) show the measured frequency response function of the assembly in the initial prestressed $\left(P_{0}\right)$ (Fig. $5(a)$ ) and the snapped $\left(P_{1}^{-}\right)$ (Fig. 5(b)) positions, respectively. The peaks in Fig. 5 are the measured resonance (or natural) frequencies. The first three theoretical and experimental natural frequencies are listed in Tables 1 and 2 for the two positions. It is observed that the natural frequencies in the snapped position are in general lower than those in the initial prestressed position. This is due to the fact that the arch is compressed more in the snapped position. The agreements between the theory and experiment in the first natural frequency, both in $P_{0}$ and $P_{1}^{-}$, are very good. The discrepancies in the other
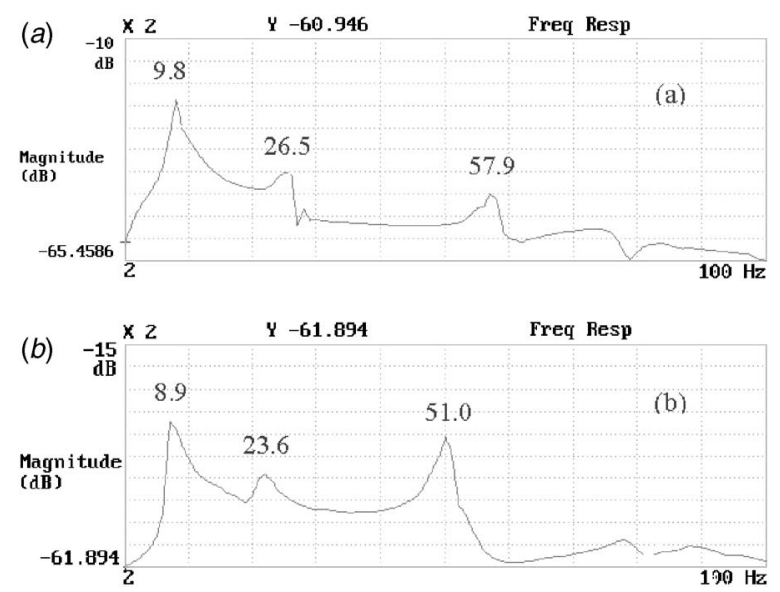

Fig. 5 Measured frequency response functions of the assembly in (a) the initial prestressed position and (b) the snapped position 
Table 1 The first three natural frequencies (theoretical and experimental) of the assembly in the initial prestressed position

\begin{tabular}{lcrr}
\hline \multicolumn{1}{c}{$n$} & 1 & 2 & 3 \\
\hline$\omega_{n}(\mathrm{~Hz})$ (theory) & 9.8 & 29.2 & 55.5 \\
$\omega_{n}(\mathrm{~Hz})$ (experiment) & 9.8 & 26.5 & 57.9 \\
Discrepancy (\%) & 0.0 & 9.2 & 4.3 \\
\hline \hline
\end{tabular}

modes are in general less than $10 \%$. These observations give us confidence that the mechanical model described in Fig. 1 and the estimated physical parameters may not be too far from the truth.

In the numerical simulation of the forced response of the assembly under the excitation of the mechanical shaker, we also need to know the damping parameters of the assembly. The damping effect comes mostly from the friction in all the joint pairs with relative motion. For simplicity we assume that the system damping can be modeled as viscous type and its effects for all modes are identical. In other words, for the damped system, Eqs. (13) and (14) are modified by adding a term $\mu \dot{\alpha}_{n}$ to the left-hand sides of the equations. To determine the damping ratio, we move the end slider (with the shaker attached) a small distance by hand and release it. The lateral displacement history at the middle point of the arch is then recorded. From the decaying rate between consecutive peaks, we can estimate the damping ratio as $5.2 \%$ [18]. The physical and dimensionless damping parameters of the arch are calculated as $\mu=2.64 \mathrm{~kg} / \mathrm{s}$ and $\mu^{*}=0.3$, respectively.

\section{Snapping of the Arch Under Harmonic End Excitation}

With the experimental setup described above, we are ready to excite the arch. After moving the shaker-mount $7 \mathrm{~mm}$ toward the arch ends, the middle point of the arch moves a distance $y-y_{0}$ $=21 \mathrm{~mm}\left(y^{*}-y_{0}^{*}=72.6\right)$ upward. The solid curve in Fig. 6 represents the lateral displacement history at the middle point of the arch when the shaker excites the assembly at the first natural frequency $9.8 \mathrm{~Hz}$. The magnetic force is estimated as $23 \mathrm{~N}$. The arch starts with zero initial velocity. After four oscillations with

Table 2 The first three natural frequencies (theoretical and experimental) of the assembly in the snapped position

\begin{tabular}{lrrr}
\hline \multicolumn{1}{c}{$n$} & 1 & 2 & 3 \\
\hline$\omega_{n}(\mathrm{~Hz})$ (theory) & 8.7 & 25.2 & 55.5 \\
$\omega_{n}(\mathrm{~Hz})$ (experiment) & 8.9 & 23.6 & 51.0 \\
Discrepancy (\%) & 2.3 & 6.3 & 8.1 \\
\hline \hline
\end{tabular}

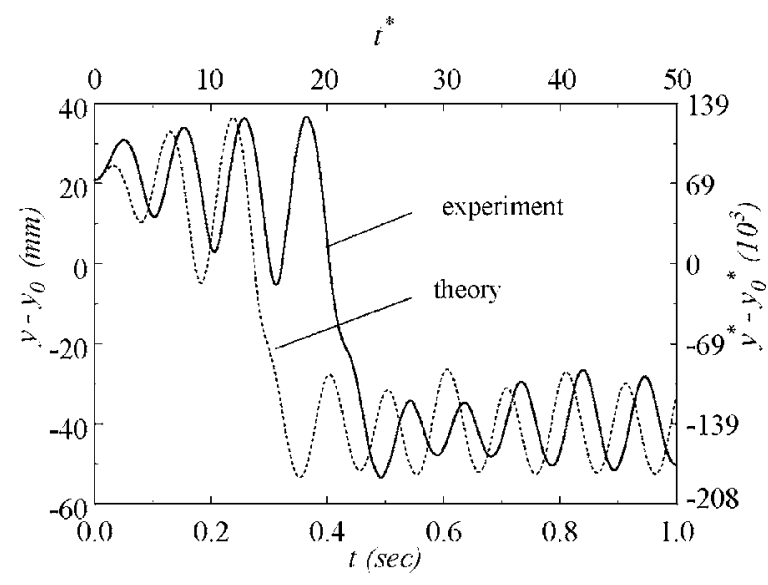

Fig. 6 Experimental (solid curve) and theoretical (dashed curve) lateral displacement history at the middle point of the arch. The arch starts at the initial prestressed position.

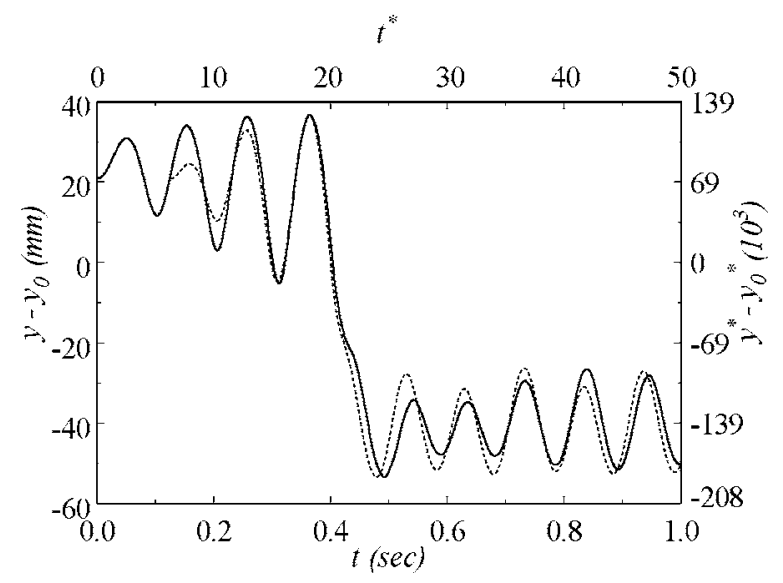

Fig. 7 Replot of Fig. 6 by shifting the numerical simulation result (dashed curve) to the right $0.13 \mathrm{~s}$ such that the instants of the last peaks before snapping are aligned

increasing amplitude, the arch snaps to the other side at time $t$ $=0.47 \mathrm{~s}$. After snapping, the arch settles to a vibration pattern superposed onto the static displacement of the snapped position. It is noted that with this excitation frequency and magnetic force, the arch will not snap back to the original position. The magnitude of the magnetic force, which is controlled by the electric current pumping through the shaker, is critical in this experiment. If the force is too small, the arch will not snap. On the other hand, if the force is too large, the arch will not remain vibrating near the snapped position. Instead it will continue to snap back and forth between the two positions. With the current experimental setup, our experience shows that there is a small range of magnetic force, approximately between $21.8 \mathrm{~N}$ and $24.6 \mathrm{~N}$, in which the arch can snap to and remain vibrating on the other side.

The dashed curve in Fig. 6 represents the numerical simulation of the action described in the experiment. One obvious discrepancy between the experiment and the theory is the time of snapping. In the numerical simulation, the arch oscillates three times before it snaps, which is compared to four oscillations in the experiment. As a consequence, in the numerical simulation the arch snaps earlier at $t=0.33 \mathrm{~s}$. This may be due to the inaccuracy in estimating the system damping. It appears that we underestimate the damping effect in the numerical simulation when the assembly starts from rest. It is recalled that we estimate the system damping by assuming it be of viscous type. In addition, the displacement amplitudes used in the logarithmic decrement technique are taken when the assembly has experienced two or three oscillations. In reality, the damping effect is due mostly to friction force in the joints. Therefore, it is possible that the damping force is larger when the assembly starts from rest than when the assembly has started moving for a while. This can be seen from the agreement of vibration amplitudes between experiment and theory, especially after the snapping action.

In Fig. 7, we ignore the discrepancy in the early stage of the transient response and focus our attention on the snapping action by shifting the dashed curve to the right $0.13 \mathrm{~s}$ such that the instants of the last peaks before snapping are aligned. We can see fairly good agreement between the theory and the experiment in the time span immediately before and after snapping.

Beside the lateral displacement at the middle point of the arch, we also record the longitudinal displacement history of the end slider $m$ (solid curves) and the shaker central core $m_{s}$ (dashed curves) when snapping occurs in Fig. 8. It is noted that the amplitude of the end slider is indeed about one-third of that of the shaker. This agrees with our expectation when we design the spring $k$ in an earlier section. It is noted that in this experiment, the central core of the shaker has a stroke of nearly $2 \mathrm{~mm}$. Appar- 


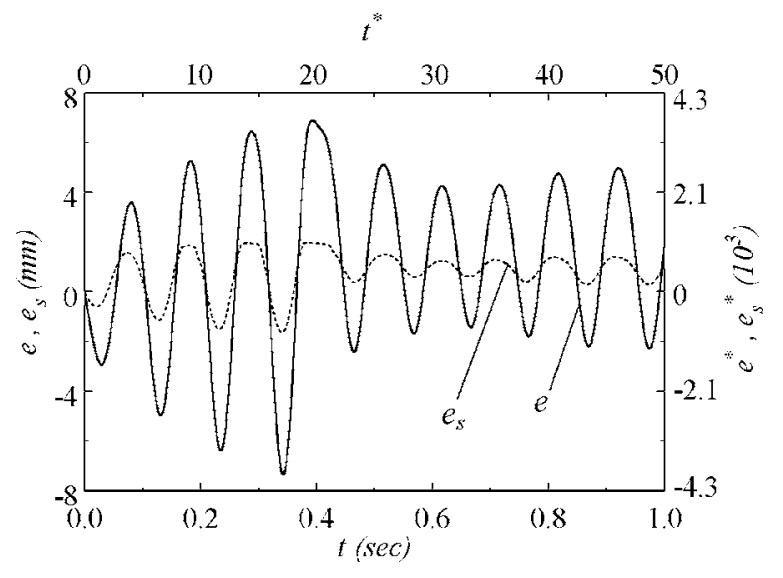

Fig. 8 The measured longitudinal displacement history of the end slider (solid curve) and the shaker (dashed curve) in the experiment described in Fig. 6

ently, the added spring $k$ reduces the mechanical impedance as seen from the shaker, as compared to the design without $k$ in [12].

When the arch is in the snapped position, it is relatively easier to snap it back to the other side by adjusting the excitation frequency of the shaker to the first natural frequency of the snapped position, i.e., $8.7 \mathrm{~Hz}$, as recorded in Fig. 9. The excitation force is $17 \mathrm{~N}$ in this case. The solid and dashed curves represent experimental result and numerical simulation, respectively. In Fig. 9, the dashed line is shifted to the right such that the instants of the last peaks before snapping back are aligned. Again, fairly good agreement between the experiment and theory is observed during the vibrant snapping action.

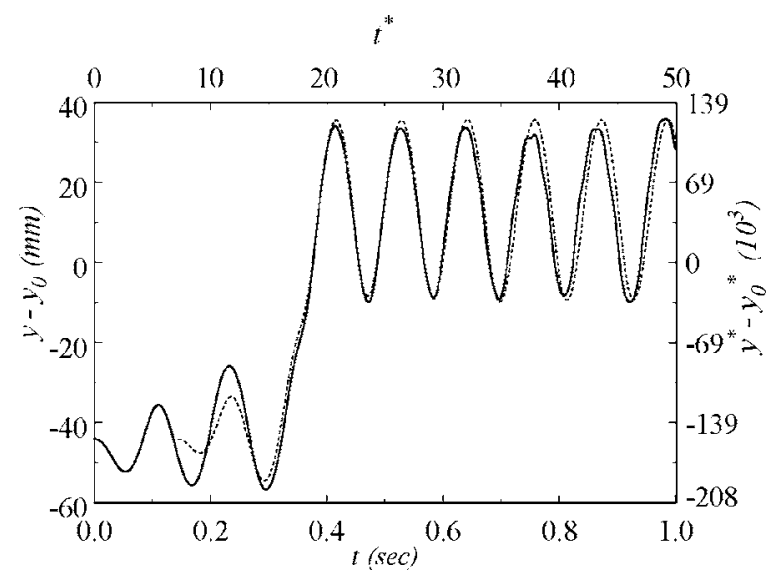

Fig. 9 Experimental (solid curve) and theoretical (dashed curve) lateral displacement history at the middle point of the arch. The arch starts at the snapped position. The dashed curve is shifted to the right $0.14 \mathrm{~s}$ such that the instants of the last peaks before snapping back are aligned.

\section{Conclusions}

In this paper we demonstrate both numerically and experimentally that it is possible to snap a pinned-pinned shallow arch back and forth between two positions at will by harmonic excitation at the end. In the experimental setup, one end of the arch is fixed in space, while the other end is attached to a shaker via a spring. The shaker-mount is first moved a small distance toward the arch to ensure that the arch possesses two stable equilibrium positions, one on each side of the base line. The spring connecting the arch slider and the mechanical shaker is carefully chosen such that a small shaker stroke can induce a large vibration amplitude of the arch. By adjusting the excitation frequency of the shaker to the first natural frequency of the assembly and carefully adjusting the magnitude of the magnetic force in the shaker, we demonstrate successfully that the arch can snap to and remain vibrating on the other side. To snap the arch back, we simply adjust the excitation frequency of the shaker to the first natural frequency of the snapped position and reduce the magnetic force. This experimental setup represents a new scheme to control a bistable device that may find application in the future.

\section{References}

[1] Timoshenko, S. P., 1935, "Buckling of Flat Curved Bars and Slightly Curved Plates," ASME J. Appl. Mech., 2, pp. 17-20.

[2] Hoff, N. J., and Bruce, V. G., 1954, "Dynamic Analysis of the Buckling of Laterally Loaded Flat Arches," J. Math. Phys. (Cambridge, Mass.), 32, pp. 276-288.

[3] Simitses, G. J., 1990, Dynamic Stability of Suddenly Loaded Structures, Springer-Verlag, New York.

[4] Chen, J.-S., and Yang, M.-R., 2007, "Vibration and Stability of a Shallow Arch Under a Moving Mass-Dashpot-Spring System," ASME J. Vibr. Acoust., 129, pp. 66-72.

[5] Thomsen, J. J., 1992, "Chaotic Vibrations of Non-Shallow Arches," J. Sound Vib., 153, pp. 239-258.

[6] Bolotin, V. V., 1964, The Dynamic Stability of Elastic Systems, Holden-Day, Inc., San Francisco.

[7] Tien, W.-M., Sri Namachchivaya, N., and Bajaj, A. K., 1994, "Non-Linear Dynamics of a Shallow Arch Under Periodic Excitation-I. 1:1 Internal Resonance," Int. J. Non-Linear Mech., 29, pp. 367-386.

[8] Tien, W.-M., Sri Namachchivaya, N., and Bajaj, A. K., 1994, "Non-Linear Dynamics of a Shallow Arch Under Periodic Excitation-II. 1:2 Internal Resonance,” Int. J. Non-Linear Mech., 28, pp. 349-366.

[9] Bi, Q., and Dai, H. H., 2000, "Analysis of Nonlinear Dynamics and Bifurcations of a Shallow Arch Subjected to Periodic Excitation With Internal Resonance," J. Sound Vib., 233, pp. 557-571.

[10] Malhotra, N., and Sri Namachchivaya, N., 1997, "Chaotic Dynamics of Shallow Arch Structures Under 1:1 Resonance,” J. Eng. Mech., 123, pp. 620-627.

[11] Malhotra, N., and Sri Namachchivaya, N., 1997, "Chaotic Dynamics of Shallow Arch Structures Under 1:2 Resonance,” J. Eng. Mech., 123, pp. 612-619.

[12] Chen, J.-S., and Yang, C.-H., "Experiment and Theory on the Nonlinear Vibration of a Shallow Arch Under Harmonic Excitation at the End," ASME Journal of Applied Mechanics, in press.

[13] Huang, N. C., 1972, "Dynamic Buckling of Some Elastic Shallow Structures Subject to Periodic Loading With High Frequency," Int. J. Solids Struct., 8, pp. 315-326.

[14] Plaut, R. H., and Hsieh, J.-C., 1985, "Oscillations and Instability of a Shallow Arch Under Two-Frequency Excitation," J. Sound Vib., 102, pp. 189-201.

[15] Blair, K. B., Krousgrill, C. M., and Farris, T. N., 1996, "Non-Linear Dynamic Response of Shallow Arches to Harmonic Forcing," J. Sound Vib., 194, pp. 353-367.

[16] Chang, D.-W., 2006, "On the Snapping of a Shallow Arch Under Harmonic Excitation at the End," MS thesis, Department of Mechanical Engineering, National Taiwan University, Taipei, Taiwan

[17] Chen, J.-S., and Lin, J.-S., 2006, "Stability of a Shallow Arch With One End Moving at Constant Speed," Int. J. Non-Linear Mech., 41, pp. 706-715.

[18] Rao, S. S., 1995, Mechanical Vibrations, 3rd ed., Addison-Wesley Publishing Company, Reading, MA 